\title{
Terahertz near-field microspectroscopy
}

\author{
J. R. Knab, ${ }^{\text {a) }}$ A. J. L. Adam, R. Chakkittakandy, and P. C. M. Planken \\ Department of Imaging Science and Technology, Faculty of Applied Sciences, Optics Research Group, \\ Delft University of Technology, Lorentzweg 1, 2628 CJ Delft, The Netherlands
}

(Received 13 May 2010; accepted 29 June 2010; published online 22 July 2010)

\begin{abstract}
Using near-field, terahertz time-domain spectroscopy (THz-TDS), we investigate how the addition of a dielectric material into a subwavelength-diameter, cylindrical waveguide affects its transmission properties. The THz electric near-field is imaged with deep subwavelength resolution as it emerges from filled and unfilled waveguides. Spectroscopic data measured for waveguides filled with polycrystalline D-tartaric acid, and with polyethylene and silicon powders, illustrate the feasibility of this approach for obtaining spectroscopic information from a tiny sample volume. (C) 2010 American Institute of Physics. [doi:10.1063/1.3467192]
\end{abstract}

Terahertz time-domain spectroscopy $(\mathrm{THz}-\mathrm{TDS})^{1}$ has emerged as an important spectroscopic tool for investigations of, for example, molecular crystals. ${ }^{2,3}$ These measurements are commonly performed on macroscopic samples in the far field, meaning that their lateral dimensions are typically much larger than the THz wavelength. The samples must be this large because the classical diffraction limit restricts the lateral sample dimensions to values larger than $\sim \lambda / 2$, which translates into a value of $150 \mu \mathrm{m}$ at a typical frequency of 1.0 THz. In practice, these samples must be considerably larger than this to avoid diffraction/edge effects. It can be advantageous to perform spectroscopic measurements on a microscopic scale, as one could measure a very small quantity of material and ensure greater sample homogeneity. To do so, however, is not trivial. One must satisfy two conditions: high spatial confinement of the field at the point of measurement and reasonably large signal-to-noise ratio in the detected signal. One way to achieve confinement of the field is by using a waveguide. ${ }^{4-8}$ For example, a parallel plate waveguide, partially filled with a thin film has been shown to be very effective for thin film far-field measurements of various systems, ${ }^{9,10}$ though additional lenses are required for in- and out-coupling of the $\mathrm{THz}$ light.

In this work, we demonstrate a different approach to waveguide THz-TDS. Free-space $\mathrm{THz}$ radiation is focused onto truncated, (sub)wavelength-diameter, cylindrical waveguides, which are completely filled with different dielectric materials. Only $\mathrm{THz}$ light that emerges from the guides and thus must have interacted with the sample is measured in the near field. We show how the waveguides can be used to resolve molecular resonances as well as the effective refractive index from a small volume of a sample, such as D-tartaric acid (DTA). Near-field spectroscopic measurements allow for an extremely high, subwavelength spatial resolution and high sensitivity for measurements of microscopic volumes.

Using near-field, terahertz time-domain spectroscopy, ${ }^{11-16}$ we acquire time- and frequency-domain images, along with corresponding spectral information for filled and unfilled waveguides (single, isolated guides, and arrays). A schematic drawing that illustrates our near-field measurement technique is shown in Fig. 1. Linearly polarized THz

${ }^{a)}$ Electronic mail: j.r.knab@tudelft.nl. light is focused onto the waveguide array, while the array itself is placed in contact with a (110)-oriented GaP electrooptic detection crystal. The THz light that emerges from the waveguides is measured in a plane immediately behind the array, pixel-by-pixel, using a counter-propagating, nearinfrared probe pulse $(\lambda=800 \mathrm{~nm}){ }^{15,16}$

A microscope image of a filled and unfilled waveguide within an array is shown in the inset of Fig. 1. The array and the single waveguide are comprised of $\sim 150 \mu \mathrm{m}$ diameter, cylindrical holes which were milled in an aluminum plate having a thickness of $\sim 180 \mu \mathrm{m}$ (waveguide volume $\sim 3.2 \mathrm{nl}$ ). The period of the array is $600 \mu \mathrm{m}$. Part of the array was filled with two different samples: polyethylene powder $\left(d_{\mathrm{avg}}=30 \mu \mathrm{m}\right.$; Mipelon XM-220, Mitsui Chemicals America, Inc.) and polycrystalline DTA (T206; SigmaAldrich Co.), a food additive. The single waveguide was filled with silicon powder $\left(d_{\max }=45 \mu \mathrm{m}\right.$; SI006015, Goodfellow Cambridge Ltd). The samples are preground using a mortar and pestle (excluding polyethylene) in order to reduce the particle size. To fill the waveguides, they are first closed on one side with Parafilm ${ }^{\circledR}$ M. The sample is then worked into the waveguides using a spatula. Samples made in this way appeared uniformly filled upon inspection with an optical microscope.

In Fig. 2, we show three images of the x-component of the THz electric near field for a DTA-filled and unfilled

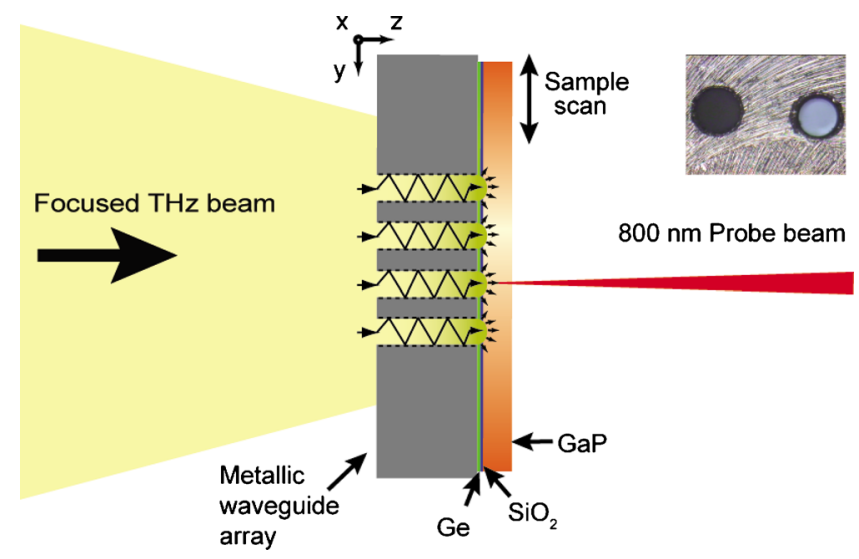

FIG. 1. (Color online) Near-field imaging and spectroscopy setup. The $\mathrm{SiO}_{2}$ and Ge layers form a high-reflection coating on the GaP detection crystal for the counter-propagating probe beam. Inset shows dielectric-filled and unfilled waveguides (left and right, respectively) within an array. 


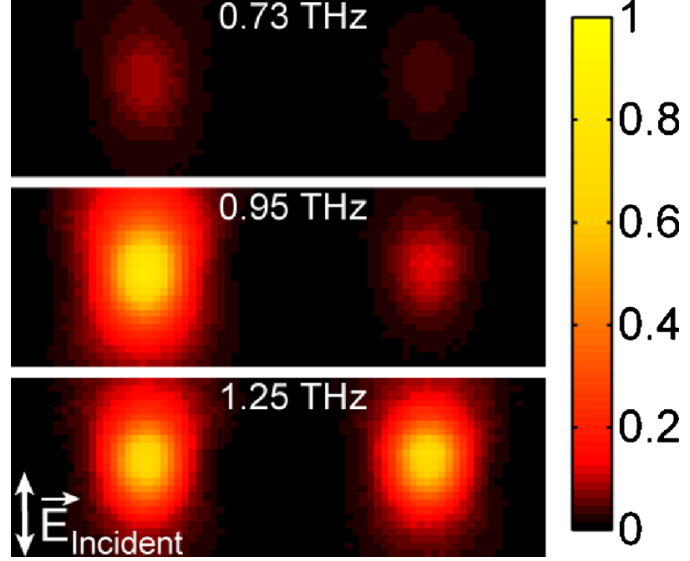

FIG. 2. (Color online) THz electric near-field, $\left|E_{x}\right|$, normalized to the incoming field, as it emerges from two adjacent waveguides within an array $(\mathrm{P}=600 \mu \mathrm{m})$. The waveguide on the left is filled with DTA while the one on the right is empty. Scan area: $1200 \times 400 \mu \mathrm{m}^{2}$. Bright (dark) color corresponds to high (low) electric field amplitude. Data were acquired in a nitrogen-purged environment.

waveguide (left and right, respectively) at three different frequencies. The two waveguides are next to each other in a $4 \times 4$ waveguide array (period $=600 \mu \mathrm{m}$ ); all data were recorded in a single measurement. The incident $\mathrm{THz}$ field is linearly polarized in the plane of the sample, vertical relative to the image shown. This image shows the canonical $\mathrm{TE}_{11}$ waveguide mode emerging from both waveguides. This is the dominant mode for a cylindrical waveguide, ${ }^{8}$ where the metal that comprises the guide is assumed to behave like a perfect conductor at $\mathrm{THz}$ frequencies. At the lower two frequencies, 0.73 and $0.95 \mathrm{THz}$, there is significantly more field emerging from the filled waveguide relative to the unfilled waveguide, while at the higher frequency, the field distributions are approximately the same. This is also illustrated in Fig. 3(a), which shows the THz electric field as a function of time from a single pixel in the middle of a polyethylene (PE) powder-filled waveguide and an adjacent, unfilled waveguide within an array. These data have been windowed to remove the etalon reflection from the $300 \mu \mathrm{m}$ thick detection crystal.

It may seem counterintuitive that filling a waveguide with a dielectric medium should increase the transmission. However, the presence of a dielectric material within a waveguide effectively reduces its cutoff frequency according to: $\nu_{c}^{\prime}=\nu_{c} / \sqrt{\varepsilon_{R}}$, where $\varepsilon_{R}$ is the real part of the dielectric permittivity and $\nu_{c}$ is the cutoff frequency. ${ }^{17-19}$ This allows more low-frequency light to be squeezed through the waveguide. In Fig. 3(b), we plot the frequency-dependent magnitude of $E_{x}$, calculated from the single-pixel data shown in Fig. 3(a), for PE, and for silicon powder in a single, isolated waveguide. In each case, the measured $\mathrm{THz}$ field was normalized to the incident field. The oscillations in the spectra originate from etalon reflections within the dielectric-filled guide. We note that the two low-frequency oscillations in the PE data $(\sim 0.7$ and $\sim 0.85 \mathrm{THz})$ are likely artifacts caused by the application of a temporal window in the time domain, which is used to remove the etalon reflection from the $300 \mu \mathrm{m}$ thick GaP detection crystal. For a high index material like silicon, the reflections, and thus the oscillations in the frequency-domain, are much more pronounced. This was verified with simulations for a single waveguide using CST
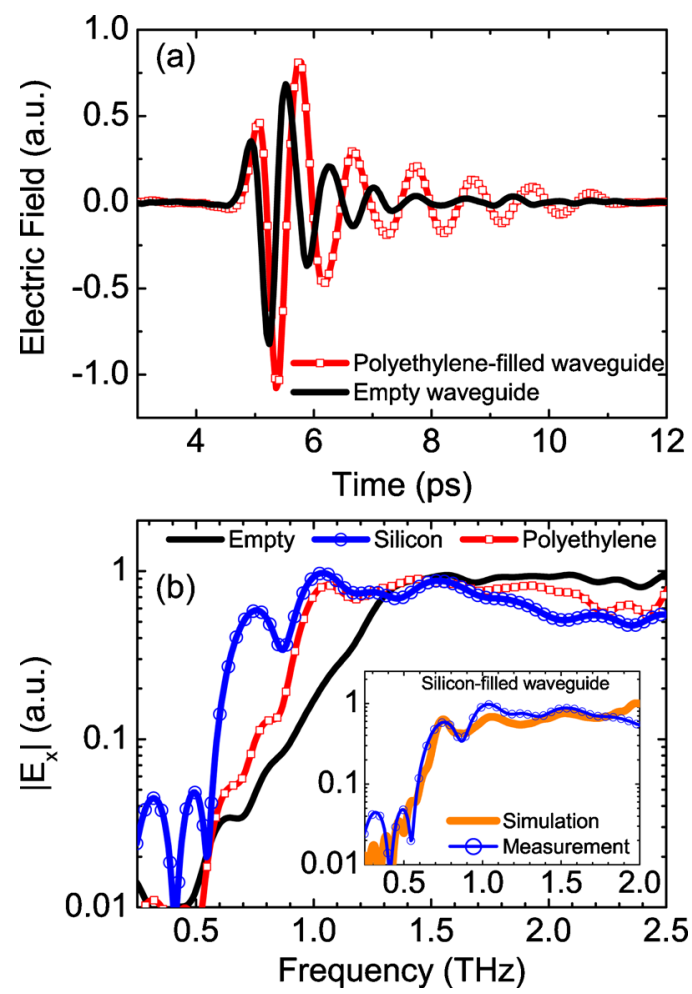

FIG. 3. (Color online) (a) $E_{x}$, measured in the near field, as it emerges from $\mathrm{PE}-$ filled and unfilled waveguides within a $4 \times 4$ array $(\mathrm{P}=600 \mu \mathrm{m}$; d=150 m). (b) $E_{x}$ spectrum, normalized to incoming field, for silicon-filled, PE-filled and empty waveguides. Inset compares spectrum for silicon-filled waveguide with simulation. Silicon data are from a single waveguide $(\mathrm{d}=150 \mu \mathrm{m})$. All data were acquired in a nitrogen-purged environment.

Microwave Studio ${ }^{\circledR}$; a comparison of a simulation for a single, silicon-filled guide and the measured data is shown in the inset of Fig. 3(b). The cutoff frequency $(\sim 1.20 \mathrm{THz}$ for the empty waveguide) clearly shifts to lower frequencies as the permittivity of the filling medium increases $\left(\varepsilon_{R_{-} \text {silicon }}\right.$ $\sim 11.7 ; \varepsilon_{R_{\perp} \mathrm{PE}} \sim 2.25$ ), thus substantially improving transmission at these frequencies. The shift in the spectrum is consistent with earlier work. ${ }^{17}$ Note that the presence of a dielectric will change the impedance of the waveguide modes. This will result in reflections at the input and output of the guide, ${ }^{8}$ reducing transmission above the cutoff frequency.

The shift in the transmission spectrum for a filled waveguide relative to an empty guide is inversely proportional to the refractive index, $n$, of the filling medium $\left(n=\sqrt{\varepsilon_{R}}\right)$. The spectral shifts for silicon and PE powders imply refractive indices of 1.81 and 1.28 , respectively. These values are considerably lower than the bulk values of $n_{\mathrm{Si}} \sim 3.42$ and $n_{\mathrm{PE}}$ $\sim 1.5$, presumably because the powder samples within the waveguides are effective media, comprised of air and the sample powders. Though the Maxwell Garnett model ${ }^{20}$ only applies to spherical particles and low filling fractions, using this approximation, we obtain a rough estimate for the filling fraction of the PE- and silicon-filled guides: $\sim 0.6$ and $\sim 0.55$, respectively.

The frequency-dependent transmission spectrum for a waveguide filled with polycrystalline DTA is shown Fig. 4(a). The spectrum of an empty, adjacent guide within the array was recorded in the same measurement and is shown for comparison. Both spectra are normalized to the incident spectrum. The DTA spectrum represents an average of several pixels near the middle of the waveguide. Several dips in 


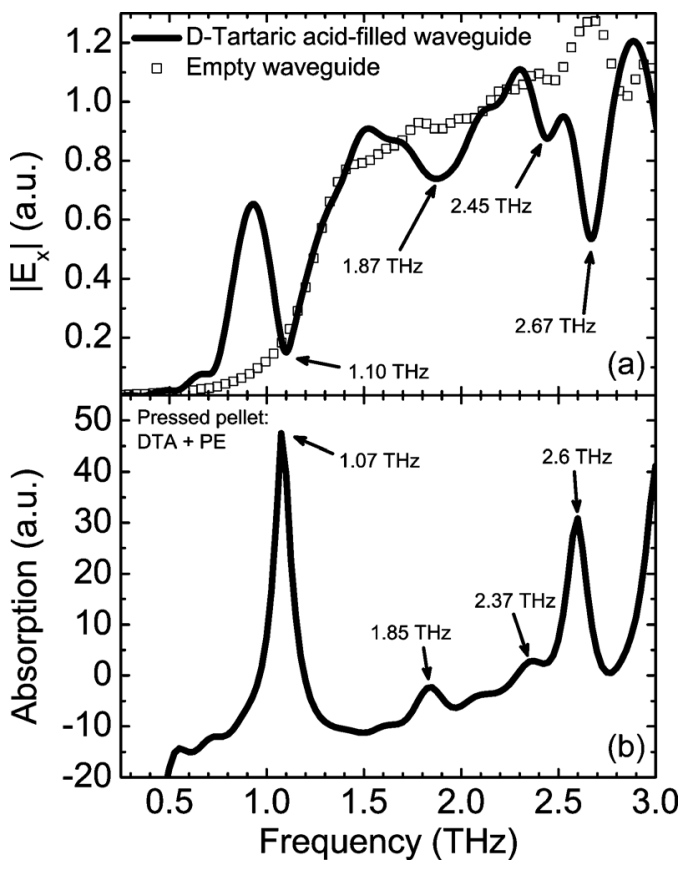

FIG. 4. (a) Near-field $E_{x}$ spectrum for DTA-filled and unfilled waveguides, which are adjacent to one another within a $4 \times 4$ array $(\mathrm{P}=600 \mu \mathrm{m}$; $\mathrm{d}=150 \mu \mathrm{m}$ ). Data were acquired in a nitrogen-purged environment. (b) Absorption spectrum for DTA+PE pressed pellet (30:70 mass ratio), recorded in quasi near field setup (see Ref. 3). The uncertainty in the position of the absorption lines in (a) and (b) is approximately $+/-50 \mathrm{GHz}$.

the spectrum are indicated, the strongest being at $1.10 \mathrm{THz}$. These are not water lines, as the measurement was performed in a nitrogen-purged environment. In fact, these spectral features are resonances corresponding to intermolecular vibrational modes. ${ }^{2}$ This is shown in Fig. 4(b), where we plot the frequency-dependent absorption for a pressed pellet, comprised of $70 \%$ PE and 30\% DTA, measured in a "quasi near field" setup. ${ }^{3}$ The positions of the four resonances in the waveguide data show very good agreement with these data, as well as with those previously reported in the literature. ${ }^{2}$

An important distinction between the DTA data shown in Fig. 4 and previous works is that here, these resonances are clearly resolved in a pure sample, which has not been mixed with a binding agent (i.e., PE powder) or otherwise manipulated. The fact that we can clearly observe resonances in such a tiny amount of material, as well as a shift in the waveguide cutoff which is proportional to the refractive index of the material, illustrates the possibility of using this technique to perform near-field, spectroscopic measurements. Using a waveguide can be advantageous because it is relatively easy to fill and one can immediately determine the refractive index. We note that for spectroscopic measurements, it is not necessary to record an entire two-dimensional image, though doing so is useful for verifying sample homogeneity. The fact that we use a truncated waveguide minimizes loss and ensures relatively high transmission. Measur- ing in the near field also provides us with the sensitivity required to measure the nonpropagating evanescent fields below the cutoff frequency. A comparable measurement in the far field, on such a tiny volume, would be almost impossible. Placing the sample into a waveguide and measuring in the near field ensures that the entire measured $\mathrm{THz}$ field has interacted with the sample.

Our preliminary results establish proof of principle for a near-field approach to waveguide TDS. Though we have only considered cylindrical waveguides in this work, different waveguide geometries, such as squares or slits, could be more advantageous. Since these are near-field measurements, the volume of the guides and thus, the sample volume, can be significantly reduced. Shorter waveguides, for example, would allow more transmission below the cutoff frequency, allowing us to decrease the waveguide diameter, and thus the sample volume, even further. ${ }^{21}$

The authors gratefully acknowledge financial support from Nederlandse Organisatie voor Wetenschappelijk Onderzoek (NWO) for a VICI grant. We also want to thank Aad Poot from TU Delft Demo for producing high-quality waveguide arrays.

${ }^{1}$ P. Y. Han and X. C. Zhang, Meas. Sci. Technol. 12, 1747 (2001)

${ }^{2}$ B. M. Fischer, H. Helm, and P. U. Jepsen, Proc. IEEE 95, 1592 (2007).

${ }^{3}$ R. Chakkittakandy, J. A. W. M. Corver, and P. C. M. Planken, Opt. Express 16, 12794 (2008).

${ }^{4}$ D. G. Cooke and P. U. Jepsen, Phys. Status Solidi A 206, 997 (2009).

${ }^{5}$ J. A. Deibel, K. Wang, M. D. Escarra, and D. M. Mittleman, Opt. Express 14, 279 (2006).

${ }^{6}$ M. Walther, M. R. Freeman, and F. A. Hegmann, Appl. Phys. Lett. 87, 261107 (2005).

${ }^{7}$ B. You, J.-Y. Lu, T.-A. Liu, J.-L. Peng, and C.-L. Pan, Appl. Phys. Lett. 96, 051105 (2010).

${ }^{8}$ G. Gallot, S. P. Jamison, R. W. McGowan, and D. Grischkowsky, J. Opt. Soc. Am. B 17, 851 (2000).

${ }^{9}$ N. Laman, S. Sree Harsha, D. Grischkowsky, and J. S. Melinger, Biophys. J. 94, 1010 (2008).

${ }^{10}$ J. S. Melinger, N. Laman, S. Sree Harsha, and D. Grischkowsky, Appl Phys. Lett. 89, 251110 (2006).

${ }^{11}$ O. Mitrofanov and J. A. Harrington, Opt. Express 18, 1898 (2010)

${ }^{12}$ A. Bitzer and M. Walther, Appl. Phys. Lett. 92, 231101 (2008).

${ }^{13}$ M. A. Seo, A. J. L. Adam, J. H. Kang, J. W. Lee, S. C. Jeoung, Q. H. Park, P. C. M. Planken, and D. S. Kim, Opt. Express 15, 11781 (2007).

${ }^{14}$ G. Zhao, R. N. Schouten, N. C. J. van der Valk, W. Th Wenckebach, and P. C. M. Planken, Rev. Sci. Instrum. 73, 1715 (2002).

${ }^{15}$ A. J. L. Adam, J. M. Brok, M. A. Seo, K. J. Ahn, D. S. Kim, J. H. Kang, Q. H. Park, M. Nagel, and P. C. M. Planken, Opt. Express 16, 7407 (2008).

${ }^{16}$ J. R. Knab, A. J. L. Adam, M. Nagel, E. Shaner, M. A. Seo, D. S. Kim, and P. C. M. Planken, Opt. Express 17, 15072 (2009).

${ }^{17}$ F. Keilmann, Int. J. Infrared Millim. Waves 2, 259 (1981).

${ }^{18}$ F. J. García de Abajo, J. J. Sáenz, I. Campillo, and J. S. Dolado, Opt. Express 14, 7 (2006)

${ }^{19}$ F. J. García-Vidal, E. Moreno, J. A. Porto, and L. Martín-Moreno, Phys. Rev. Lett. 95, 103901 (2005).

${ }^{20}$ J. C. Maxwell Garnett, Philos. Trans. R. Soc. London, Ser. A 203, 385 (1904)

${ }^{21}$ L. Guestin, A. J. L. Adam, J. R. Knab, M. Nagel, and P. C. M. Planken, Opt. Express 17, 17412 (2009). 